\title{
INEQUALITIES FOR LINEAR COMBINATIONS OF ORDER STATISTICS FROM RESTRICTED FAMILIES
}

\author{
By Richard E. Barlow ${ }^{1}$ and Frank Proschan
}

\section{University of California, Berkeley, and Boeing Scientific Research Laboratories}

1. Introduction. In this paper we present some results of theoretical interest concerning order statistics and their spacings from certain restricted families of positive random variables. Applications to life testing are discussed in a separate paper [Barlow and Proschan (in process)].

For a specified continuous distribution $G$ for which $G(0)=0$, we consider the family $\mathcal{F}$ of distributions such that for $F$ in $F$ and $F(0)=0, G^{-1} F$ is starshaped or convex on the support of $F$. Distributions related in this way by convexity have been studied by Van Zwet (1964). It is known that $F(0)=0$, $G(0)=0$, and $G^{-1} F$ convex imply $G^{-1} F$ starshaped. [Bruckner and Ostrow (1962).]

If $G$ is the exponential distribution, then $G^{-1} F$ convex where finite is equivalent to $F$ having an increasing failure rate (i.e., $F$ is IFR). $G^{-1} F$ starshaped is equiv alent to $F$ having an increasing failure rate average (i.e., $F$ is IFRA) [Birnbaum, Esary, and Marshall (1965)]. $G^{-1} F$ concave on [0, $)$ is equivalent to $F$ having decreasing failure rate (i.e., $F$ is DFR).

If $G$ is the uniform distribution, then $G^{-1} F$ convex on the support of $F$ is equivalent to $F$ having an increasing density. If $F(G)$ denotes the gamma distribution with shape parameter $\alpha(\beta)$ with $\alpha>\beta$, then $G^{-1} F$ is convex on $[0, \infty)$ [Van $Z_{w e t}$ (1964)]. The Weibull family is similarly ordered, as may be readily verified.

Comparisons for linear combinations of expected values of order statistics from $F$ and $G$ are obtained when $G^{-1} F$ is starshaped. In addition, stochastic comparisons for linear combinations of order statistics are obtained when $G^{-1} F$ is convex as well as when $G^{-1} F$ is starshaped.

Specializing to the case where $G$ is the exponential distribution and $F$ is IFR or IFRA, stochastic comparisons are made for the "total time on test," which is of interest in life testing. Bounds on the expected values of order statistics are also obtained for this case.

Finally, we investigate the preservation of certain class properties under the operation of taking order statistics.

2. Preliminaries. Throughout this paper we adopt the following notation and assumptions. Let $X(Y)$ have distribution $F(G)$. We assume that $F(0)=0=$ $G(0)$, and that $F$ and $G$ are continuous. We also assume that the support of $F$ is

\footnotetext{
Received 8 April 1966.

${ }^{1}$ Research partially supported by the Office of Naval Research Contract Nonr-3656(18) with the University of California. Work completed while the author was a Visiting Research Associate at Boeing Scientific Research Laboratories.
} 
an interval, possibly infinite, and that $G$ is strictly increasing on its support. We use $\bar{F}$ for $1-F$ and $\bar{G}$ for $1-G$.

A function $\phi$ is starshaped on $[0, b), 0<b \leqq \infty$, if $\phi(\alpha x) \leqq \alpha \phi(x)$ for $0 \leqq \alpha \leqq 1,0 \leqq x<b$ (or equivalently, if $\phi(x) / x$ is increasing for $x$ in $[0, b)$ ); and $\phi$ is convex on $(a, b),-\infty \leqq a<b \leqq \infty$, if $\phi[\alpha x+(1-\alpha) y] \leqq \alpha \phi(x)+$ $(1-\alpha) \phi(y)$ for $0 \leqq \alpha \leqq 1, a<x, y<b$. Then on $[0, b)$, convex $\phi$ such that $\phi(0) \leqq 0$ are starshaped.

The following properties of IFR (DFR) distributions will be needed [cf. Barlow and Proschan (1965), Chapter II]. If $F$ is IFR (DFR) and

then

$$
\begin{aligned}
G(x) & =0, & & x<0, \\
& =1-e^{-x}, & & x \geqq 0,
\end{aligned}
$$

(i) $G^{-1} F$ is convex (concave) where finite (on $\left.[0, \infty)\right)$;

(ii) $F_{u}(x)=[F(x+u)-F(u)] / \bar{F}(u)$ is increasing (decreasing) in $u \geqq 0$ for all $x \geqq 0$ whenever the denominator is nonzero;

(iii) $G^{-1} F\left(F^{-1} G\right)$ is starshaped where defined (on $\left.[0, \infty)\right)$;

(iv) $[\bar{F}(x)]^{x^{-1}}$ is decreasing (increasing) in $x \geqq 0$.

Let $X_{1 n} \leqq \cdots \leqq X_{n n}\left(Y_{1 n} \leqq \cdots \leqq Y_{n n}\right)$ denote an ordered sample of size $n$ from $F(G)$; define $X_{0 n} \equiv 0\left(Y_{0 n} \equiv 0\right)$. We drop the second subscript when there is no danger of confusion. We use the term increasing (decreasing) for nondecreasing (nonincreasing). We use the notation $\geqq_{\mathrm{st}}\left(\varliminf_{\mathrm{st}}\right.$ ) for "stochastically greater than" ("stochastically less than") and = to".

Finally, we use repeatedly the relationship $\left(G^{-1} F\left(X_{1}\right), \cdots, G^{-1} F\left(X_{n}\right)\right)={ }_{\text {st }}$ $\left(Y_{1}, \cdots, Y_{n}\right)$.

3. Inequalities in the case of starshapedness. In this section we consider pairs of distributions $F$ and $G$ such that $G^{-1} F$ is starshaped on the support of $F$. We shall obtain a stochastic comparison between linear combinations of order statistics $X_{1 n} \leqq \cdots \leqq X_{n n}$ from $F$ and $Y_{1 n} \leqq \cdots \leqq Y_{n n}$ from $G$. To do this we first present some basic inequalities for starshaped functions. For further discussion and extension of Lemmas 3.1 and 3.3, see Barlow, Marshall, and Proschan (in process). We shall find it convenient to define $\bar{A}_{i}=\sum_{j=i}^{n} a_{j}$, where throughout the $a_{j}$ represent real numbers.

LEMMA 3.1.

$$
\phi\left(\sum_{i=1}^{n} a_{i} x_{i}\right) \leqq \sum_{i=1}^{n} a_{i} \phi\left(x_{i}\right)
$$

for all starshaped $\phi$ on $[0, b)$ and all $0 \leqq x_{1} \leqq \cdots \leqq x_{n}<b$ for which $0 \leqq \sum_{i=1}^{n} a_{i} x_{i}<b$ if and only if there exists $k(1 \leqq k \leqq n)$ such that $0 \leqq \bar{A}_{1} \leqq$ $\cdots \leqq \bar{A}_{k} \leqq 1$, and when $k<n, \bar{A}_{k+1}=\cdots=\bar{A}_{n}=0$.

REMARK. Note that if (3.1) holds for all starshaped $\phi$ on $[0, b]$ and all $0 \leqq x_{1} \leqq \cdots \leqq x_{n}<b$, then it holds for all starshaped $\phi$ on $\left[0, b^{\prime}\right)$ and all $0 \leqq x_{1} \leqq \cdots \leqq x_{n}<b^{\prime}$, where $b^{\prime}$ is any positive number. A similar remark may be made in connection with Lemmas 3.3, 4.1 and 4.3. 
Proof. Sufficiency. Assume $0 \leqq \bar{A}_{1} \leqq \cdots \leqq \bar{A}_{k} \leqq 1$, and when $k<n$, $\bar{A}_{k+1}=\cdots=\bar{A}_{n}=0$. Then $a_{i} \leqq 0$ for $i=1,2, \cdots, k-1,0 \leqq a_{k} \leqq 1$, and when $k<n, a_{i}=0$ for $i=k+1, \cdots, n$. Using the identity $\sum_{1}^{n} a_{i} x_{i} \equiv$ $\sum_{1}^{n} \bar{A}_{i}\left(x_{i}-x_{i-1}\right)$, where $x_{0} \equiv 0$, we conclude that $0 \leqq \sum_{1}^{n} a_{i} x_{i} \leqq x_{k}$. Thus $\phi\left(x_{k}\right) / x_{k} \geqq \phi\left(x_{i}\right) / x_{i}$ for $i=1, \cdots, k-1$, and $\phi\left(x_{k}\right) / x_{k} \geqq \phi\left(\sum a_{i} x_{i}\right) / \sum a_{i} x_{i}$. Hence $\left\{\sum_{1}^{k-1}\left(-a_{i}\right) x_{i}+\sum_{1}^{n} a_{i} x_{i}\right\} \phi\left(x_{k}\right) / x_{k} \geqq \sum_{1}^{k-1}\left(-a_{i}\right) \phi\left(x_{i}\right)+\phi\left(\sum a_{i} x_{i}\right)$, or $a_{k} \phi\left(x_{k}\right) \geqq \sum_{1}^{k-1}\left(-a_{i}\right) \phi\left(x_{i}\right)+\phi\left(\sum a_{i} x_{i}\right)$.

Necessity. Let $\phi(x)=x^{2}, 0=x_{1}=\cdots=x_{i-1}$, and $x_{i}=\cdots=x_{n}=b^{\prime}<b$. Then (3.1) implies $\left(\sum_{j=i}^{n} a_{j}\right)^{2} \leqq \sum_{j=i}^{n} a_{j}$, so that $0 \leqq \bar{A}_{i} \leqq 1$ for $i=1,2, \cdots, n$.

Next we shall show that $\bar{A}_{j}>0$ implies $\bar{A}_{j-1} \leqq \bar{A}_{j}$. To see this, let $0=x_{1}=\cdots=x_{j-2}<x_{j-1}<x_{j}=x_{j+1}=\cdots=x_{n}<b$. Then $\sum_{1}^{n} a_{i} x_{i} \equiv$ $\sum_{1}^{n} \bar{A}_{i}\left(x_{i}-x_{i-1}\right)=\bar{A}_{j-1} x_{j-1}+\bar{A}_{j}\left(x_{j}-x_{j-1}\right)$. Fix $x_{j}$ and choose $x_{j-1}$ and $z$ sufficiently small so that $x_{j-1}<z<x_{j}$ and $\sum_{1}^{n} a_{i} x_{i}>z$. Let

$$
\begin{aligned}
\phi_{z}(x) & =0, \quad x<z, \\
& =x, \quad x \geqq z,
\end{aligned}
$$

a starshaped function. From (3.1),

$$
\phi_{z}\left(\sum_{1}^{n} a_{i} x_{i}\right)=\bar{A}_{j-1} x_{j-1}+\bar{A}_{j}\left(x_{j}-x_{j-1}\right) \leqq \bar{A}_{j} x_{j} .
$$

This implies $\bar{A}_{j-1}-\bar{A}_{j} \leqq 0$.

Finally, if each $\bar{A}_{i}$ is zero, the proof is complete. If not, let $k$ denote the largest subscript $i$ such that $\bar{A}_{i}>0$. Assume that $\bar{A}_{j+1}=0$ for $j<k-1$. We shall show that this implies $\bar{A}_{i}=0$ for $i \leqq j$. Let $x_{j}<z \leqq x_{j+1}$ and $x_{k}$ be so large that $\sum_{1}^{n} a_{i} x_{i}=\sum_{1}^{k} \bar{A}_{i}\left(x_{i}-x_{i-1}\right)>z$. Then

$$
\phi_{z}\left(\sum_{1}^{n} a_{i} x_{i}\right)=\sum_{1}^{n} a_{i} x_{i} \leqq \sum_{j+1}^{n} a_{i} x_{i}
$$

which impies $\sum_{1}^{j} a_{i} x_{i}=\sum_{1}^{j} \bar{A}_{i}\left(x_{i}-x_{i-1}\right)+\bar{A}_{j+1} x_{j} \leqq 0$. This in turn implies $\bar{A}_{i}=0$ for $i=1,2, \cdots, j$ since $\bar{A}_{j+1}=0$ and $0 \leqq \bar{A}_{i} \leqq 1$. $\square$

THEOREM 3.2. Let $G^{-1} F$ be starshaped on the support of $F, F(0)=0=G(0)$. If there exists $k(1 \leqq k \leqq n)$ such that

$$
0 \leqq \bar{A}_{1} \leqq \bar{A}_{2} \leqq \cdots \leqq \bar{A}_{k} \leqq 1, \text { and when } k<n, \bar{A}_{k+1}=\cdots=\bar{A}_{n}=0
$$

then

$$
F\left(\sum_{1}^{n} a_{i} X_{i}\right) \leqq_{\mathrm{st}} G\left(\sum_{1}^{n} a_{i} Y_{i}\right) .
$$

Proof. By Lemma 3.1,

$$
G^{-1} F\left(\sum_{1}^{n} a_{i} X_{i}\right) \leqq \sum_{1}^{n} a_{i} G^{-1} F\left(X_{i}\right)={ }_{\text {st }} \sum_{1}^{n} a_{i} Y_{i} .
$$

Theorem 3.2 can be used to obtain conservative lower tolerance limits [Barlow, Proschan (1966)].

To obtain a reverse inequality to that of $(3.2)$ we need the following lemma:

Lemma 3.3.

$$
\phi\left(\sum_{1}^{n} a_{i} x_{i}\right) \geqq \sum_{1}^{n} a_{i} \phi\left(x_{i}\right)
$$


for all starshaped $\phi$ on $[0, b)$ and all $0 \leqq x_{1} \leqq \cdots \leqq x_{n}<b$ for which $0 \leqq \sum_{1}^{n} a_{i} x_{i}<b$ if and only if there exists $k(1 \leqq k \leqq n)$ such that

$$
\bar{A}_{1} \geqq \bar{A}_{2} \geqq \cdots \geqq \bar{A}_{k} \geqq 1 ; \quad \bar{A}_{k+1}=\cdots=\bar{A}_{n}=0,
$$

or equivalently, $a_{i} \geqq 0$ for $1 \leqq i \leqq k ; a_{k} \geqq 1$ and $a_{i}=0$ for $i=k+1, \cdots, n$.

Proof. Sufficiency. We may assume $x_{1}>0$. Hence

$$
\phi\left(\sum_{1}^{k} a_{i} x_{i}\right) / \sum_{1}^{k} a_{i} x_{i} \geqq \phi\left(x_{i}\right) / x_{i}
$$

for $i=1,2, \cdots, k$, since $\phi$ is starshaped and $\sum_{1}^{k} a_{i} x_{i} \geqq x_{k}$. It follows that $\sum_{1}^{k} a_{i} x_{i} \phi\left(\sum_{1}^{k} a_{i} x_{i}\right) / \sum_{1}^{k} a_{i} x_{i} \geqq \sum_{1}^{k} a_{i} \phi\left(x_{i}\right)$, yielding (3.3).

Necessity. From the proof of necessity in Lemma 3.1 we see that for each $i$, either $\bar{A}_{i} \leqq 0$ or $\bar{A}_{i} \geqq 1$. First we claim $\bar{A}_{i} \leqq 0$ implies $\bar{A}_{i+1} \leqq 0$, and hence $\bar{A}_{i+1} \geqq 1$ implies $\bar{A}_{i} \geqq 1$. To see this, let $0=x_{1}=\cdots=x_{i-1}<x_{i}<z<$ $x_{i+1}=\cdots=x_{n}$. Choose $\left(x_{i+1}-x_{i}\right)$ sufficiently small so that

$$
\sum_{1}^{n} a_{i} x_{i}=\bar{A}_{i} x_{i}+\bar{A}_{i+1}\left(x_{i+1}-x_{i}\right) \leqq x_{i} .
$$

We can do this since by assumption $\bar{A}_{i} \leqq 0$. Hence by $(3.3), \phi_{z}\left(\sum_{1}^{n} a_{i} x_{i}\right)=$ $0 \geqq \bar{A}_{i+1} x_{i+1}$, which implies $\bar{A}_{i+1} \leqq 0$. Thus we have shown that for some $k(0 \leqq k \leqq n), \bar{A}_{1} \geqq 1, \cdots, \bar{A}_{k} \geqq 1 ; \bar{A}_{k+1} \leqq 0, \cdots, \bar{A}_{n} \leqq 0$.

We claim that we cannot have $\bar{A}_{i} \geqq 1$ and $\bar{A}_{j}<0$ for $j>i$. Suppose this were the case. Choose $0=x_{1}=\cdots=x_{i-1}<z<x_{i}=x_{i+1}=\cdots=x_{j-1}<$ $x_{j}=\cdots=x_{n}<b$, and $\left(x_{j}-x_{i}\right)$ and $z$ in such a manner that

$$
0<\sum_{1}^{n} \bar{A}_{i}\left(x_{i}-x_{i-1}\right)=\bar{A}_{i} x_{i}+\bar{A}_{j}\left(x_{j}-x_{i}\right)<z .
$$

Then by (3.3)

$$
\phi_{z}\left[\sum_{1}^{n} a_{i} x_{i}\right]=0 \geqq \bar{A}_{i} x_{i}+\bar{A}_{j}\left(x_{j}-x_{i}\right)>0,
$$

which is a contradiction. It follows that $\bar{A}_{k+1}=\cdots=\bar{A}_{n}=0$.

Next we shall show that $\bar{A}_{i+1} \geqq 1$ implies $\bar{A}_{i} \geqq \bar{A}_{i+1}$. To see this, let $0=x_{1}=\cdots=x_{i-1}<x_{i}<z<x_{i+1}=\cdots=x_{n}<b$. Then

$$
\sum_{1}^{n} a_{i} x_{i}=\bar{A}_{i} x_{i}+\bar{A}_{i+1}\left(x_{i+1}-x_{i}\right) \geqq x_{i+1},
$$

which implies by (3.3) that

$$
\phi_{z}\left(\sum_{1}^{n} a_{i} x_{i}\right)=\bar{A}_{i} x_{i}+\bar{A}_{i+1}\left(x_{i+1}-x_{i}\right) \geqq \bar{A}_{i+1} x_{i+1} .
$$

This implies $\bar{A}_{i} \geqq \bar{A}_{i+1} \cdot \square$

From Lemma 3.3 we obtain

THEOREM 3.4. Let $G^{-1} F$ be starshaped on the support of $F$ and $F(0)=0=G(0)$. Let $a_{i} \geqq 0$ for $i=1,2, \cdots, n$, and $a_{n} \geqq 1$. Then

$$
F\left(\sum_{1}^{n} a_{i} X_{i}\right) \geqq_{\mathrm{st}} G\left(\sum_{1}^{n} a_{i} Y_{i}\right) .
$$

Proof. By assumption, the support of $F$ is an interval, say $[0, b]$. When $\sum_{1}^{n} a_{i} X_{i}>b$, then $F\left(\sum_{1}^{n} a_{i} X_{i}\right)=1 \geqq G\left(\sum_{1}^{n} a_{i} Y_{i}\right)$. Consider outcomes for 
which $\sum_{1}^{n} a_{i} X_{i}<b$. Apply Lemma 3.3 to obtain

$$
G^{-1} F\left(\sum_{1}^{n} a_{i} X_{i}\right) \geqq \sum_{1}^{n} a_{i} G^{-1} F\left(X_{i}\right) .
$$

Since $\sum_{1}^{n} a_{i} G^{-1} F\left(X_{i}\right)={ }_{\text {st }} \sum_{1}^{n} a_{i} Y_{i}$, (3.4) holds.

Theorem 3.4 can be used to obtain conservative upper tolerance limits [Barlow, Proschan (1966)].

The following useful results concerning order statistics are immediate consequences of the theory of total positivity. For definitions, notation, and theory see Karlin (1964).

LEMma 3.5. Let $F_{i, n}$ denote the distribution of the $i$ th order statistic in a sample of size $n$ from a continuous distribution $F$ defined on $(-\infty, \infty)$. Suppose $g(x)$ changes sign $k$ times and

$$
h(i, n)=\int_{-\infty}^{\infty} g(x) d F_{i, n}(x)
$$

converges absolutely. Then

(i) $h(i, n)$ changes sign at most $k$ times as a function of $i=1,2, \cdots$, for fixed $n$, and changes sign at most $k$ times as a function of $n=1,2, \cdots$, for fixed $i$. Furthermore, if $h(i, n)$ changes sign exactly $k$ times as a function of $i(n)$, then $h(i, n)$ must have the same (opposite) arrangement of signs in $i(n)$ as $g(x)$ where $x, i$, and $n$ traverse their respective domains from left to right.

(ii) $h(n-i, n)$ changes sign at most $k$ times as a function of $n=1,2, \cdots$; if $h(n-i, n)$ actually does change sign in $n$ exactly $k$ times, then the changes occur in the same order as those of $g(x)$.

Proof. Since $F$ is continuous we may write

$$
\begin{aligned}
h(i, n) & =n\left(\begin{array}{c}
n \\
i-1
\end{array}\right) \int_{-\infty}^{\infty} g(x) F^{i-1}(x) \bar{F}^{n-i}(x) d F(x) \\
& \equiv \int_{-\infty}^{\infty} g(x) K(i, n ; x) d F(x) .
\end{aligned}
$$

It is easily verified that $K(i, n ; x)$ is totally positive of order $\infty \quad\left(T P_{\infty}\right)$ in $i=1,2, \cdots$ and $-\infty<x<\infty$. Also $K(i, n ; x)$ is reverse regular of order $\infty\left(R R_{\infty}\right)$ in $n=1,2, \cdots$ and $-\infty<x<\infty$ and $T P_{\infty}$ in $n=1,2, \cdots$ and $-\infty<x<\infty$. The results follow from the variation diminishing property of totally positive functions. $\square$

Theorem 3.6. Let $G^{-1} F$ be starshaped on the support of $F$ and $F(0)=0=G(0)$. Then $E X_{\text {in }} / E Y_{\text {in }}$ is

(i) decreasing in $i$,

(ii) increasing in $n$,

(iii) $E X_{n-i, n} / E Y_{n-i, n}$ is decreasing in $n$.

Proof. Let $\phi(x)=G^{-1} F(x)$. Since $\phi$ is starshaped, then for arbitrary $c \geqq 0$, $x-c \phi(x)$ changes sign at most once, and from positive to negative values if at all. Hence

$$
h(i, n)=\int_{0}^{\infty}[x-c \phi(x)] d F_{i n}(x)=E X_{i n}-c E Y_{\text {in }}
$$

changes sign at most once in $i=1,2, \cdots(n=1,2, \cdots)$, and from positive 
(negative) to negative (positive) values if at all by Lemma 3.5. Therefore $\left[E X_{i n} / E Y_{i n}\right]-c$ changes sign at most once in $i(n)$ and from positive (negative) to negative (positive) values if at all. Since $c \geqq 0$ is arbitrary, the ratio $E X_{i n} / E Y_{i n}$ is decreasing (increasing) in $i(n)$.

Again by Lemma 3.5, $h(n-i, n)$ changes sign at most once in $n=1,2, \cdots$ and from positive to negative values if at all. By a similar argument as above we see that $E X_{n-i, n} / E Y_{n-i, n}$ is decreasing in $n$.

Choosing $G$ to be the uniform distribution we see from Theorem 3.6 that $(n+1) E X_{i n} / i$ is decreasing in $i$ and increasing in $n$, where $X_{1 n} \leqq \cdots \leqq X_{n n}$ are order statistics from $F$, a distribution with increasing density. Choosing $G$ to be the exponential distribution, we see that $E X_{i n} / \sum_{j=1}^{i}(n-j+1)^{-1}$ is decreasing in $i$ and increasing in $n$, where $X_{1 n} \leqq \cdots \leqq X_{n n}$ are order statistics from $F$, an IFRA distribution.

By using Theorem 3.6, bounds on $E X_{\text {in }}$ can be obtained as follows. Note that

$$
E X_{i i} / E Y_{i i} \leqq E X_{i n} / E Y_{i n} \leqq E X_{1, n-i+1} / E Y_{1, n-i+1},
$$

the first inequality from Theorem 3.6 (ii), the second from Theorem 3.6 (iii). Now if $E X=E Y=\theta$, then

$$
\theta E Y_{\text {in }} / E Y_{i i} \leqq E X_{\text {in }} \leqq \theta E Y_{\text {in }} / E Y_{1, n-\imath+1} .
$$

To obtain an application of Theorem 3.6 (iii) we choose $G$ uniform on $[0,1]$ and $F$ such that $f$ is increasing on the support of $F$. Then we immediately have $(n+1) E X_{n-i, n} /(n-i)$ decreasing in $n \geqq i+1(i=0,1, \cdots)$. This is a strengthening of the monotonicity result of Corollary 4 of Marshall and Proschan (1965) which implies as a special case that $E X_{n n} / n$ is decreasing in $n$ whenever the underlying distribution $F$ satisfies $F\left(0^{-}\right)=0$.

We will need the following lemma:

Lemma 3.7. If $\beta_{i} / \alpha_{i}$ is increasing in $i(1 \leqq i \leqq n), \alpha_{i}>0$, and $\beta_{i} \geqq 0$, then

(i) $\sum_{1}^{r} \beta_{i} / \sum_{1}^{r} \alpha_{i}$ and

(i') $\sum_{1}^{r}(n-i+1)\left(\beta_{i}-\beta_{i-1}\right) / \sum_{1}^{r}(n-i+1)\left(\alpha_{i}-\alpha_{i-1}\right)$ are increasing in $r(1 \leqq r \leqq n)$;

in particular,

(ii) $\sum_{1}^{r} \beta_{i} / \sum_{1}^{n} \beta_{i} \leqq \sum_{1}^{r} \alpha_{i} / \sum_{1}^{n} \alpha_{i}$ and

(ii') $\sum_{1}^{r}(n-i+1)\left(\beta_{i}-\beta_{i-1}\right) / \sum_{1}^{i} \beta_{i} \leqq \sum_{1}^{r}(n-i+1)\left(\alpha_{i}-\alpha_{i-1}\right) / \sum_{1}^{n} \alpha_{i}$;

(iii) $\sum_{1}^{n} a_{i} \beta_{i} / \sum_{1}^{n} \beta_{i} \leqq \sum_{1}^{n} a_{i} \alpha_{i} / \sum_{1}^{n} \alpha_{i}$ and

(iii') $\sum_{1}^{n} a_{i}(n-i+1)\left(\beta_{i}-\beta_{i-1}\right) / \sum_{1}^{n} \beta_{i} \leqq \sum_{1}^{n} a_{i}(n-i+1)\left(\alpha_{i}-\alpha_{i-1}\right) / \sum_{1}^{n} \alpha_{i}$ if $a_{1} \geqq a_{2} \geqq \cdots \geqq a_{n}$.

Proof.

(i) Define $\psi(0)=0, \psi\left(\alpha_{1}+\cdots+\alpha_{i}\right)=\beta_{1}+\cdots+\beta_{i}(1 \leqq i \leqq n)$. Define $\psi(x)$ elsewhere on $\left[0, \alpha_{1}+\cdots+\alpha_{n}\right]$ by linear interpolation between successive points defined above. Note that

$$
\begin{aligned}
{\left[\psi\left(\alpha_{1}+\cdots+\alpha_{i}\right)-\psi\left(\alpha_{1}+\cdots+\alpha_{i-1}\right)\right] } & \\
& {\left[\left(\alpha_{1}+\cdots+\alpha_{i}\right)-\left(\alpha_{1}+\cdots+\alpha_{i-1}\right)\right]^{-1}=\beta_{i} / \alpha_{i} }
\end{aligned}
$$


is increasing in $i$, so that $\psi$ is a convex function on $\left[0, \alpha_{1}+\cdots+\alpha_{n}\right]$. Since $\psi(0)=0, \psi$ is also starshaped, i.e. $\psi(x) / x$ is increasing in $x$. Hence

$$
\psi\left[\sum_{1}^{r} \alpha_{i}\right] / \sum_{1}^{r} \alpha_{\imath}=\sum_{1}^{r} \beta_{i} / \sum_{1}^{r} \alpha_{i}
$$

is increasing in $r$.

(i') Write $\sum_{1}^{r}(n-i+1)\left(\alpha_{i}-\alpha_{i-1}\right)=\alpha_{1}+\cdots+\alpha_{r}+\overbrace{\alpha_{r}+\cdots+\alpha_{r}}^{r}$ and similarly for $\sum_{1}^{r}(n-i+1)\left(\beta_{i}-\beta_{i-1}\right)$. The result is geometrically obvious.

(ii) and (ii') are immediate consequences of (i) and $\left(\mathrm{i}^{\prime}\right)$.

(iii) Let $\sum_{1}^{n} a_{i}\left[\beta_{i} / \sum \beta_{i}-\alpha_{i} / \sum \alpha_{i}\right]=\sum_{1}^{n} a_{i} d_{i}$, and note that

$$
\sum a_{i} d_{i}=\left(a_{1}-a_{2}\right) d_{1}+\left(a_{2}-a_{3}\right)\left(d_{1}+d_{2}\right)+\cdots+a_{n}\left(d_{1}+\cdots+d_{n}\right) \text {. }
$$

Since $a_{i-1}-a_{i} \geqq 0, i=1,2, \cdots, n-1, d_{1}+\cdots+d_{i} \leqq 0(i=1,2, \cdots$, $n-1)$, and $d_{1}+\cdots+d_{n}=0$, we conclude that $\sum_{1}^{n} a_{i} d_{i} \leqq 0$.

(iii') is proved similarly.

To prove the next result, we need to introduce the following concepts:

Definition. A sequence $\underline{a}=\left(a_{1}, \cdots, a_{n}\right)$ is said to majorize a sequence $\underline{b}=\left(b_{1}, \cdots, b_{n}\right) \quad\left(\right.$ written $\underline{a}>\underline{b}$ ) if $a_{1} \geqq \cdots \geqq a_{n}, b_{1} \geqq \cdots \geqq b_{n}$, and $\sum_{1}^{r} a_{i} \geqq \sum_{1}^{r} b_{i}$ for $r=1, \cdots, n-1$, while $\sum_{1}^{n} a_{i}=\sum_{1}^{n} b_{i}$.

This definition differs slightly from that of Hardy, Littlewood, Pólya (1959), p. 45, but corresponds to the usage of Beckenbach and Bellman (1961), p. 30.

Definition. If a differentiable function $H\left(z_{1}, \cdots, z_{n}\right)$ satisfies

$$
\left(z_{i}-z_{j}\right)\left(\partial H / \partial z_{i}-\partial H / \partial z_{j}\right) \geqq 0
$$

for all $z, i, j$, then $H$ is said to satisfy the Schur condition.

We shall use the following theorem (see Ostrowski (1952)):

TheOREm 3.8. (Schur, Ostrowski). Assume $H$ is defined for $z_{1} \geqq \cdots \geqq z_{n}$ and has partial derivatives. Then $H(\underline{z}) \geqq H\left(\underline{z}^{\prime}\right)$ for all $\underline{z}>\underline{z}^{\prime}$ if and only if $H(\underline{z})$ satisfies the Schur condition.

Theorem 3.9. Let $G^{-1} F$ be starshaped on the support of $F, F(0)=G(0)=0$, and $E X=E Y$. Then

(i) $\sum_{1}^{r} E Y_{i} / \sum_{1}^{r} E X_{i}$ and $\sum_{1}^{r}(n-i+1) E\left(Y_{i}-Y_{i-1}\right) / \sum_{1}^{r}(n-i+1)$ $E\left(X_{i}-X_{i-1}\right)$ are increasing in $r(1 \leqq r \leqq n)$;

(ii) $\left(E Y_{n}, E Y_{n-1}, \cdots, E Y_{1}\right)>\left(E X_{n}, E X_{n-1}, \cdots, E X_{1}\right)$ and $\sum_{1}^{r}(n-i+1) E\left(X_{i}-X_{i-1}\right) \geqq \sum_{1}^{r}(n-i+1) E\left(Y_{i}-Y_{i-1}\right)$ for $1 \leqq$ $r \leqq n$;

(iii) $H\left(E Y_{n}, E Y_{n-1}, \cdots, E Y_{1}\right) \geqq H\left(E X_{n}, E X_{n-1}, \cdots, E X_{1}\right)$ if $H$ is a Schur function;

(iv) $\sum_{1}^{n} a_{i}(n-i+1) E\left(X_{i}-X_{i-1}\right) \geqq \sum_{1}^{n} a_{i}(n-i+1) E\left(Y_{i}-Y_{i-1}\right)$ if $a_{1} \geqq a_{2} \geqq \cdots \geqq a_{n}$.

Proof. Since $E Y_{i} / E X_{i}$ is increasing in $i$ by Theorem 3.6, (i) follows from Lemma 3.7 (i). Since $E X=E Y$, (ii) follows from Lemma 3.7 (ii). (iii) follows from (ii) and Theorem 3.8. (iv) follows from Lemma 3.7 (iii). 
The following result presented in Hardy, Littlewood, and Pólya (1959), p. 89 , is used to obtain Corollary 3.11 .

TheOREM 3.10. If $\phi$ is convex on the interval $I$ and $\underline{x}>\underline{y}$, where $x_{1}, \cdots, x_{n}$, $y_{1}, \cdots, y_{n}$ belong to $I$, then $\sum_{1}^{n} \phi\left(x_{i}\right) \geqq \sum_{1}^{n} \phi\left(y_{i}\right)$.

Conollary 3.11. Let $G^{-1} F$ be starshaped on the support of $F, F(0)=0=G(0)$, $\int_{0}^{\infty} x d F(x)=\int_{0}^{\infty} x d G(x)$, and $\psi$ be convex. Then $\sum_{1}^{n} \psi\left(E Y_{i}\right) \geqq \sum_{1}^{n} \psi\left(E X_{i}\right)$.

Proof. The result follows immediately from Theorem 3.9 (ii) and Theorem 3.10 .

The following theorem is obtained in Marshall, Olkin, and Proschan (1966).

THEOREM 3.12. If $G^{-1} F$ is starshaped on the support of $F$, then

(i) $\left(X_{n} / \sum X_{i}, \cdots, X_{1} / \sum X_{i}\right) \prec_{\text {st }}\left(Y_{n} / \sum Y_{i}, \ldots, Y_{1} / \sum Y_{i}\right){ }_{j}$

where $\left(U_{1}, \cdots, U_{n}\right) \prec_{\mathrm{st}}\left(V_{1}, \cdots, V_{n}\right)$ means $\sum_{i=1}^{n} U_{i} \leqq_{\mathrm{st}} \sum_{i=1}^{j} V_{i}$ for $j=1, \cdots, n-1$, while $\sum_{i=1}^{n} U_{i}={ }_{\text {st }} \sum_{i=1}^{n} V_{i}$;

(ii) $H\left(X_{n} / \sum X_{i}, \cdots, X_{1} / \sum X_{i}\right) \leqq_{\mathrm{st}} H\left(Y_{n} / \sum Y_{i}, \cdots, Y_{1} / \sum Y_{i}\right)$

if $H$ is a Schur function;

(iii) $\sum_{1}^{r}(n-i+1)\left(X_{i}-X_{i-1}\right) / \bar{X} \geqq_{\mathrm{st}} \sum_{1}^{r}(n-i+1)\left(Y_{i}-Y_{i-1}\right) / \bar{Y}$

(iv) $\left(n^{-1} \sum_{1}^{n} X_{i}^{2}-\bar{X}^{2}\right) / \bar{X}^{2} \leqq_{\mathrm{st}}\left(n^{-1} \sum_{1}^{n} Y_{i}^{2}-\bar{Y}^{2}\right) / \bar{Y}^{2}$.

(v) If, in addition, $a_{1} \geqq \cdots \geqq a_{n}$, then

$$
\sum_{1}^{n} a_{i} X_{i} / \bar{X} \geqq{ }_{\mathrm{st}} \sum_{1}^{n} a_{i} Y_{i} / \bar{Y}
$$

and

$$
\sum_{1}^{n} a_{i}(n-i+1)\left(X_{i}-X_{i-1}\right) / \bar{X} \geqq_{\mathrm{st}} \sum_{1}^{n} a_{i}(n-i+1)\left(Y_{i}-Y_{i-1}\right) / \bar{Y}
$$

where $\bar{X}=\sum_{1}^{n} X_{i} / n$ and $\bar{Y}=\sum_{1}^{n} Y_{i} / n$.

Proof. By definition $G^{-1} F\left(X_{i}\right) / X_{i}=Y_{i}^{\prime} / X_{i}$ is increasing in $i$. Hence (i) follows from Lemma 3.7 (i). (ii) follows from (i) and Theorem 3.8. (iii) is a consequence of Lemma 3.7 (ii). (iv) follows from (ii) where

$$
H\left(z_{1}, \cdots, z_{n}\right)=n^{-1} \sum_{1}^{n} z_{i}^{2}-1 \text {. }
$$

(v) follows from Lemma 3.7 (iii).

4. Inequalities in the case of convexity. In this section we consider pairs of distributions $F$ and $G$ such that $G^{-1} F$ is convex on the support of $F$ and $F(0)=0=G(0)$. This is a strengthening of the starshapedness hypothesis of Section 3. Our first result has applications to conservative lower tolerance limits [Barlow and Proschan (1966)]. We shall need the following inequality which is of independent interest. See Barlow, Marshall, and Proschan (in process) for further discussion and extensions. Alternative proofs of Lemmas 4.1 and 4.3 can be given using Theorem 9 of Hardy, Littlewood, and Pólya (1929).

LEMMA 4.1. (i)

$$
\phi\left(\sum_{1}^{n} a_{i} x_{i}\right)-\phi(0) \leqq \sum_{1}^{n} a_{i}\left[\phi\left(x_{i}\right)-\phi(0)\right]
$$

for all convex $\phi$ on $[0, b)$ and all $0 \leqq x_{1} \leqq \cdots \leqq x_{n}<b$ for which $0 \leqq$ $\sum_{i=1}^{n} a_{i} x_{i}<b$ if and only if $0 \leqq \bar{A}_{i} \leqq 1$ for $i=1,2, \cdots, n$. 
(ii) If (4.1) holds for all $\underline{a}$ satisfying $0 \leqq \bar{A}_{i} \leqq 1, i=1, \cdots, n$, then $\phi$ is convex on $[0, b)$.

Proof. (i) Sufficiency. First assume $\phi(0)=0$ and $0 \leqq \bar{A}_{i} \leqq 1$ for $i=1, \cdots, n$. Then

$$
\begin{aligned}
\phi\left(\sum_{1}^{n} a_{i} x_{\imath}\right)=\phi( & \left.\sum_{1}^{n} \bar{A}_{\imath}\left(x_{i}-x_{i-1}\right)\right) \\
& =\sum_{j=1}^{n}\left[\phi\left(\sum_{i=1}^{j} \bar{A}_{\imath}\left(x_{i}-x_{i-1}\right)\right)-\phi\left(\sum_{i=1}^{j-1} \bar{A}_{\imath}\left(x_{\imath}-x_{i-1}\right)\right)\right]
\end{aligned}
$$

(where $\sum_{i=1}^{0} \equiv 0$ ). Since the $\bar{A}_{j}$ are $\leqq 1$, the last expression is

$$
\begin{aligned}
& \leqq \sum_{j=1}^{n} \bar{A}_{j}\left[\phi\left(\sum_{i=1}^{j-1} \bar{A}_{i}\left(x_{i}-x_{i-1}\right)+\left(x_{j}-x_{j-1}\right)\right)-\phi\left(\sum_{i=1}^{j-1} \bar{A}_{i}\left(x_{i}-x_{i-1}\right)\right)\right] \\
& \leqq \sum_{j=1}^{n} \bar{A}_{j}\left[\phi\left(\sum_{i=1}^{j-1}\left(x_{i}-x_{i-1}\right)+\left(x_{j}-x_{j-1}\right)\right)-\phi\left(\sum_{i=1}^{j-1}\left(x_{i}-x_{i-1}\right)\right)\right] \\
& =\sum_{j=1}^{n} \bar{A}_{j}\left[\phi\left(x_{j}\right)-\phi\left(x_{j-1}\right)\right]=\sum_{j=1}^{n} a_{j} \phi\left(x_{j}\right) .
\end{aligned}
$$

Note that if we let $\psi(x)=\phi(x)-\phi(0)$ where $\phi$ is convex, then $\psi$ is convex and $\psi(0)=0$. Hence (4.1) holds for all convex $\phi$ on $[0, b)$.

(i) Necessity. Next assume (4.1) holds. Choose $\phi(x)=x^{2}$ and $0=x_{1}=\cdots=$ $x_{j-1} ; x_{j}=\cdots=x_{n}=b$. Then (4.1) implies $\left(\sum_{j}^{n} a_{i}\right)^{2} \leqq \sum_{j}^{n} a_{\imath}$, so that $0 \leqq \bar{A}_{j} \leqq 1$.

(ii) Now suppose (4.1) holds for all $\underline{a}$ satisfying $0 \leqq \bar{A}_{i} \leqq 1$ for $i=1,2$, $\cdots, n$. Then $\phi(x)-\phi(0)$ is convex on $[0, b)$ directly from the definition of convexity. Hence $\phi$ is convex on $[0, b)$.

We may now prove

THEOREM 4.2. Let $G^{-1} F$ be convex on the support of $F, F(0)=0=G(0)$, and $0 \leqq \bar{A}_{i} \leqq 1$ for $i=1, \cdots, n$. Then

$$
F\left(\sum_{1}^{n} a_{i} X_{i}\right) \leqq_{\mathrm{st}} G\left(\sum_{1}^{n} a_{i} Y_{i}\right)
$$

or equivalently,

$$
F\left[\sum_{1}^{n} \bar{A}_{i}\left(X_{i}-X_{i-1}\right)\right] \leqq_{\mathrm{st}} G\left[\sum_{1}^{n} \bar{A}_{\imath}\left(Y_{i}-Y_{i-1}\right)\right]
$$

Proof. Using Theorem 4.1, we have

$$
G^{-1} F\left(\sum_{1}^{n} a_{i} x_{i}\right) \leqq \sum_{1}^{n} a_{\imath} G^{-1} F\left(X_{i}\right)={ }_{\text {st }} \sum_{1}^{n} a_{i} Y_{i} .
$$

Thus (4.2) follows.

The equivalence of (4.3) and (4.2) follows from the identity $\sum_{1}^{n} a_{2} x_{i}=$ $\sum_{1}^{n} \bar{A}_{i}\left(x_{i}-x_{i-1}\right)$.

For specified $G$, the distribution of $G\left(\sum_{1}^{n} a_{i} Y_{i}\right)$ may be determined. Theorem 4.2 may then be used to obtain a conservative lower tolerance limit for distributions $F$ for which $G^{-1} F$ is convex [Barlow and Proschan (1966)].

To obtain a reversal of inequality (4.2) we need Theorem 4.4 below. To prove Theorem 4.4 we state the following result:

Lemma 4.3.

$$
\phi\left(\sum_{1}^{n} a_{i} x_{i}\right)-\phi(0) \geqq \sum_{1}^{n} a_{i}\left[\phi\left(x_{i}\right)-\phi(0)\right]
$$


for all convex $\phi$ on $[0, b)$ and all $0 \leqq x_{1} \leqq \cdots \leqq x_{n}<b$ for which $0 \leqq \sum_{1}^{n} a_{i} x_{i}<$ $b$ if and only if

$$
\bar{A}_{1} \geqq 1, \quad \bar{A}_{2} \geqq 1, \cdots, \bar{A}_{k} \geqq 1, \quad \bar{A}_{k+1} \leqq 0, \cdots, \bar{A}_{n} \leqq 0
$$

for some $k(0 \leqq k \leqq n)$.

Proof. Sufficiency. We shall prove the result for convex $\phi$ satisfying $\phi(0)=0$. The more general result then follows immediately.

First suppose $\sum_{1}^{n} a_{i} x_{i} \leqq x_{k}$. Then

$$
\begin{aligned}
& \sum_{1}^{k}\left(\bar{A}_{i}-1\right)\left(x_{i}-x_{i-1}\right)\left[\phi\left(x_{i}\right)-\phi\left(x_{i-1}\right)\right] /\left(x_{i}-x_{i-1}\right) \\
&+\left(x_{k}-\sum_{1}^{n} a_{i} x_{i}\right)\left[\phi\left(x_{k}\right)-\phi\left(\Sigma a_{i} x_{i}\right)\right] /\left(x_{k}-\Sigma a_{i} x_{i}\right) \\
& \leqq \sum_{k+1}^{n}\left(-\bar{A}_{i}\right)\left(x_{i}-x_{i-1}\right)\left[\phi\left(x_{i}\right)-\phi\left(x_{i-1}\right)\right] /\left(x_{i}-x_{i-1}\right),
\end{aligned}
$$

since (a) each ratio on the left is less than every ratio on the right by convexity, and (b) the sum of the coefficients on the left, $\sum_{1}^{k}\left(\bar{A}_{i}-1\right)\left(x_{i}-x_{i-1}\right)+$ $\left(x_{k}-\sum_{1}^{n} a_{i} x_{i}\right)$, equals the sum of the coefficients on the right, $\sum_{k+1}^{n}\left(-\bar{A}_{i}\right)$. $\left(x_{i}-x_{i-1}\right)$, and (c) every coefficient is positive. After simplification, the inequality reduces to the desired result.

Next suppose $\sum_{1}^{n} a_{i} x_{i}>x_{k}$. Then rewrite the inequality above as

$$
\begin{aligned}
\sum_{1}^{k}\left(\bar{A}_{i}-1\right)\left(x_{i}-x_{i-1}\right)\left[\phi\left(x_{i}\right)-\phi\left(x_{i-1}\right)\right] /\left(x_{i}-x_{i-1}\right) \\
\leqq\left\{\left[\phi\left(\Sigma a_{i} x_{i}\right)-\phi\left(x_{k}\right)\right] /\left(\Sigma a_{i} x_{i}-x_{k}\right)\right\}\left(\Sigma a_{i} x_{i}-x_{k}\right) \\
\quad+\sum_{k+1}^{n}\left(-\bar{A}_{i}\right)\left(x_{i}-x_{i-1}\right)\left[\phi\left(x_{i}\right)-\phi\left(x_{i-1}\right)\right] /\left(x_{i}-x_{i-1}\right) .
\end{aligned}
$$

The desired result follows by the same arguments as before.

Necessity. Next assume (4.4) holds for all convex $\phi$. Choose $\phi(x)=x^{2}, 0=$ $x_{1}=\cdots=x_{j-1} ; x_{j}=\cdots=x_{n}=b^{\prime}<b$. Then (4.5) implies $\left(\sum_{j}^{n} a_{i}\right)^{2} \geqq$ $\sum_{j}^{n} a_{i}$. Thus $\bar{A}_{j}$ is either $\geqq 1$ or $\leqq 0$.

Now we show that $\bar{A}_{i} \leqq 0$ implies $\bar{A}_{i+1} \leqq 0$. Choose $0=x_{1}=\cdots=x_{i-1}<$ $x_{i}=z<x_{i+1}=\cdots=x_{n} \leqq b^{\prime}$, and

$$
\begin{aligned}
& \phi(x)=0 \quad \text { for } \quad x<z \\
& =x-z \quad \text { for } \quad x \geqq z .
\end{aligned}
$$

Suppose $\bar{A}_{i} \leqq 0$ and $\bar{A}_{i+1}>0$. Choose $0<x_{i}=z<x_{i+1} \leqq b^{\prime}$ so that $0 \leqq$ $\sum_{1}^{n} a_{i} x_{i}=\bar{A}_{i} x_{i}+\bar{A}_{i+1}\left(x_{i+1}-x_{i}\right) \leqq z$. Thus $\phi\left(\Sigma a_{i} x_{i}\right)=0 \geqq \bar{A}_{i+1}\left(x_{i+1}-x_{i}\right)$ by hypothesis, which contradicts $\bar{A}_{i+1}>0$.

Finally, assume $\bar{A}_{i+1} \geqq 1$. Then $\bar{A}_{i}$ cannot be $\leqq 0$ by the result just obtained. Therefore, $\bar{A}_{i} \geqq 1$. The proof of necessity is now complete. $\square$

Using Lemma 4.3 we may now prove

TheOREM 4.4. Let $G^{-1} F$ be convex on the support of $F, F(0)=0=G(0)$, and for some $k(0 \leqq k \leqq n), \bar{A}_{i} \geqq 1, i=1, \cdots, k$, while $\bar{A}_{i} \leqq 0, i=k+1, \cdots, n$. Then

$$
F\left(\sum_{1}^{n} a_{i} X_{i}\right) \geqq_{\mathrm{s} 1} G\left(\sum_{1}^{n} a_{i} Y_{i}\right),
$$


or equivalently,

$$
F\left[\sum_{1}^{n} \bar{A}_{i}\left(X_{i}-X_{i-1}\right)\right] \geqq_{\mathrm{st}} G\left[\sum_{1}^{n} \bar{A}_{i}\left(Y_{i}-Y_{i-1}\right)\right] .
$$

Proof. Theorem 4.4 follows from Lemma 4.3 in the same way that Theorem 4.2 follows from Lemma 4.1 .

Next we obtain a comparison involving expected values of the order statistics rather than a stochastic comparison of the order statistics themselves.

Theorem 4.5. $G^{-1} F$ be convex on the support of $F, F(0)=0=G(0), a_{i} \geqq 0$ for $i=1, \cdots, n$, and $\sum_{1}^{n} a_{i} \leqq 1$. Then

$$
F\left(\sum_{1}^{n} a_{i} E X_{i}\right) \leqq G\left(\sum_{1}^{n} a_{i} E Y_{i}\right) .
$$

Proof. First using Lemma 4.1 and then Jensen's inequality, we have

$$
G^{-1} F\left(\sum_{1}^{n} a_{i} E X_{i}\right) \leqq \sum_{1}^{n} a_{i} G^{-1} F\left(E X_{i}\right) \leqq \sum_{1}^{n} a_{i} E G^{-1} F\left(X_{i}\right)=\sum_{1}^{n} a_{i} E Y_{i}
$$

We thus obtain (4.7).

This result was noted by Van Zwet (1964) for the case $a_{i}=1$ and $a_{j}=0$ for $j \neq i$, without the requirement that $F(0)=G(0)=0$. We use (4.7) in Section 6 to obtain bounds on $\sum_{1}^{n} a_{i} E X_{i}$.

As another application of Theorem 4.2, we obtain the following inequality on weighted sums of spacings.

Theorem 4.6. Let $G^{-1} F$ be convex on the support of $F, F(0)=0=G(0)$, $\theta=E X=E Y$, and $\bar{A}_{i} \geqq 1$ for $i=1, \cdots, r$. Then

$$
P_{F}\left\{\sum_{1}^{r} \bar{A}_{i}\left(X_{i}-X_{i-1}\right) \geqq x\right\} \geqq P_{G}\left\{\sum_{1}^{r} \bar{A}_{i}\left(Y_{i}-Y_{i-1}\right) \geqq x\right\}
$$

for $x \leqq \theta \min \left(\bar{A}_{1}, \cdots, \bar{A}_{r}\right)$.

Proof. For $c \geqq\left[\min \left(\bar{A}_{1}, \cdots, \bar{A}_{r}\right)\right]^{-1}$, by Theorem 4.5

$$
F\left[\sum_{1}^{r} c \bar{A}_{i}\left(X_{i}-X_{i-1}\right)\right] \geqq_{\mathrm{st}} G\left[\sum_{1}^{r} c \bar{A}_{i}\left(Y_{i}-Y_{i-1}\right)\right] .
$$

It follows that

$$
P_{F}\left\{F\left[\sum_{1}^{r} c \bar{A}_{i}\left(X_{i}-X_{i-1}\right)\right] \geqq F(\theta)\right\} \geqq P_{G}\left\{G\left[\sum_{1}^{r} c \bar{A}_{i}\left(Y_{i}-Y_{i-1}\right)\right] \geqq F(\theta)\right\} .
$$

By Theorem 7.1 of Barlow and Marshall (1964), p. 1256, $F(\theta) \leqq G(\theta)$. It follows that

$P_{F}\left\{F\left[\sum_{1}^{r} c \bar{A}_{i}\left(X_{i}-X_{i-1}\right)\right] \geqq F(\theta)\right\} \geqq P_{G}\left\{G\left[\sum_{1}^{r} c \bar{A}_{i}\left(Y_{i}-Y_{i-1}\right)\right] \geqq G(\theta)\right\}$, implying

$$
P_{F}\left\{\sum_{1}^{r} \bar{A}_{i}\left(X_{i}-X_{i-1}\right) \geqq \theta / c\right\} \geqq P_{G}\left\{\sum_{1}^{r} \bar{A}_{i}\left(Y_{i}-Y_{i-1}\right) \geqq \theta / c\right\} .
$$

Setting $x=\theta / c \leqq \theta \min \left(\bar{A}_{1}, \cdots, \bar{A}_{n}\right)$, we obtain the desired conclusion.

5. Inequalities when one distribution is the exponential. We now specialize to the case $G(x)=1-e^{-x}$ for $x \geqq 0$. The following results are motivated by the observation that in this case the normalized spacings $(n-i+1)\left(Y_{i n}-Y_{i-1, n}\right)$ 
are independent and identically distributed for $i=1,2, \cdots, n$ and $n \geqq 1$. Thus we might expect that the spacings $(n-i+1)\left(X_{i n}-X_{i-1, n}\right)$ would exhibit certain monotonicity properties for distributions $F$ such that $G^{-1} F$ is convex where finite (concave on $[0, \infty)$ ). Such distributions $F$ are IFR (DFR).

Theorem 5.1. If $F$ is IFR (DFR) with $F(0)=0$, then $(n-i+1)$. $\left(X_{\text {in }}-X_{i-1, n}\right)$ is stochastically increasing (decreasing) in $n \geqq i$ for fixed $i$.

Proof. Assume $F$ is IFR. Let $F_{i n}(x)=P\left[X_{i n} \leqq x\right]$ and $F_{u}(x)=$ $[F(x+u)-F(u)] / \bar{F}(u)$. Then

$$
\begin{aligned}
& P\left[(n-i)\left(X_{i+1, n}-X_{i n}\right)>x\right] \\
& \quad=\int_{0}^{\infty}\left\{\bar{F}_{u}[x /(n-1)]\right\}^{n-i} d F_{i n}(u) \leqq \int_{0}^{\infty}\left\{\bar{F}_{u}[x /(n+1-i)]\right\}^{n+1-i} d F_{i n}(u),
\end{aligned}
$$

since $[\bar{F}(t)]^{t^{-1}}$ is decreasing in $t$ for $F$ IFR. Also since $\bar{F}_{u}(x)$ is decreasing in $u$ for $F$ IFR and $F_{i n}(x) \leqq F_{i, n+1}(x)$ for all $F$, we have by the lemma on p. 52 of Barlow and Proschan (1965),

$$
\begin{aligned}
\int_{0}^{\infty}\left\{\bar{F}_{u}[x /(n+1-i)]\right\}^{n+1-i} d F_{i n}(u) & \leqq \int_{0}^{\infty}\left\{\bar{F}_{u}[x /(n+1-i)]\right\}^{n+1-i} d F_{i, n+1}(u) \\
= & P\left[(n+1-i)\left(X_{i+1, n+1}-X_{i, n+1}\right)>x\right] .
\end{aligned}
$$

All inequalities are reversed when $F$ is DFR.

Corollary 5.2. If $F$ is IFR (DFR) and $F(0)=0$, then $(n-i+1)$. $\left(X_{\text {in }}-X_{i-1, n}\right)$ is stochastically decreasing (increasing) in $i=1,2, \cdots, n$ for fixed $n$.

Proof. Assume $F$ is IFR. First we shall show that $(n-1)\left(X_{2 n}-X_{1 n}\right) \leqq_{\text {st }}$ $n X_{1 n}$. Given $X_{1 n}, X_{2 n}-X_{1 n}$ is the minimum of $n-1$ random variables each stochastically less than $X_{1 n}$. Hence $X_{2 n}-X_{1 n} \leqq_{\text {st }} X_{1, n-1}$. By Theorem 5.1, $(n-1) X_{1, n-1} \leqq{ }_{\text {st }} n X_{1 n}$, so that $(n-1)\left(X_{2 n}-X_{1 n}\right) \leqq{ }_{\text {st }} n X_{1 n}$. The result follows by repeated conditioning.

An analogous argument applies in the DFR case.

Next we obtain results concerning "total time on test" when successive observations are taken from an IFRA (DFRA) distribution. For example, if $n$ items are put on life test and experimentation is terminated at the time of the $r$ th failure (censored sampling), then $T_{r n}=\sum_{1}^{r}(n-i+1)\left(X_{i n}-X_{i-1, n}\right)$ denotes the total time on test. This statistic has been extensively studied and applied in the case of the exponential distribution by Epstein and Sobel (1953) and Epstein $(1960 \mathrm{a}, \mathrm{b})$. The best estimate for the mean $\theta$ in the exponential case is $\hat{\theta}_{r n}=$ $T_{r n} / r$.

Theorem 5.3. Let $F$ be IFRA (DFRA), $F(0)=0$, and $E X=\theta$. Then

(i) $\hat{\theta}_{r, n}(\underline{X}) / \bar{X} \equiv \sum_{1}^{r}(n-i+1)\left(X_{i}-X_{i-1}\right) / r \bar{X} \geqq_{\mathrm{st}}\left(\leqq_{\mathrm{st}}\right)$

$$
\sum_{1}^{r}(n-i+1)\left(Y_{i}-Y_{i-1}\right) / r \bar{Y} \equiv \hat{\theta}_{r, n}(\underline{Y}) / \bar{Y}
$$

(ii) $E \hat{\theta}_{r, n}$ is decreasing (increasing) in $r$ so that $E \hat{\theta}_{r, n} \geqq$ (ฏ) $\theta$;

(iii) $\sum_{1}^{n} a_{i}(n-i+1) E\left(X_{i}-X_{i-1}\right) \leqq(\geqq) \theta \sum_{1}^{n} a_{i}$ if $a_{1} \geqq \cdots \geqq a_{n}$.

Proof. (i) follows from Theorem 3.12 (iii). (ii) follows from Theorem 3.9) (i). (iii) follows from Theorem 3.9 (iv). 
Note that when $F$ is IFR we can assert

$$
H\left(n E X_{1},(n-1) E\left(X_{2}-X_{1}\right), \cdots, E\left(X_{n}-X_{n-1}\right)\right) \geqq H(\theta, \theta, \cdots, \theta)
$$

when $H$ is a Schur function.

TheOREM 5.4. Let $F$ be IFR (DFR) and $F\left(0^{-}\right)=0$. Then $T_{r n}=$ $\sum_{1}^{r}(n-i+1)\left(X_{i n}-X_{i-1, n}\right)$ is stochastically increasing (decreasing) in $n \geqq r$.

Proof. Assume $F$ is IFR. The proof is by induction on $r$. By Theorem 5.1 the result is true for $r=1$.

Now assume the theorem is true for $r-1$. Note that

$$
\begin{aligned}
P_{F}\left[T_{r n}>x\right] & =\int_{0}^{\infty} P_{F_{u / n}}\left[T_{r-1, n-1}>x-u\right] d_{u} P\left[n X_{1 n} \leqq u\right] \\
& \leqq \int_{0}^{\infty} P_{F_{u / n}}\left[T_{r-1, n}>x-u\right] d_{u} P\left[n X_{1 n} \leqq u\right]
\end{aligned}
$$

by the induction assumption since $F_{u / n}$ is IFR.

Next note that if $X_{1} \leqq \cdots \leqq X_{n}$ are order statistics from any distribution $F$, then $P\left[X_{2}+\cdots+X_{r}+(n-r) X_{r}>x \mid X_{1}=w\right]$ is increasing in $w$. This is a consequence of the following two facts:

(i) Given $X_{1}=w, X_{2}, \cdots, X_{n}$ are order statistics of a sample of size $n-1$ from the conditional distribution $P[X \leqq x \mid X>w]$, where $X$ has distribution $F$.

(ii) $P[X>x \mid X>w]$ is increasing in $w$.

It follows that $P_{F_{u / n}}\left[T_{r-1, n}>x-u\right]$ is increasing in $u$ for any distribution $F$. Hence

$$
\begin{aligned}
\int_{0}^{\infty} & P_{F_{u / n}}\left[T_{r-1, n}>x-u\right] d_{u} P\left[n X_{1 n} \leqq u\right] \\
& \leqq \int_{0}^{\infty} P_{F_{u / n}}\left[T_{r-1, n}>x-u\right] d_{u} P\left[(n+1) X_{1, n+1} \leqq u\right] \\
& \leqq \int_{0}^{\infty} P_{F_{u /(n+1)}}\left[T_{r-1, n}>x-u\right] d_{u} P\left[(n+1) X_{1, n+1} \leqq u\right] \\
& =P\left[T_{r, n+1}>x\right] .
\end{aligned}
$$

The last inequality follows from the fact that if $F$ is $\operatorname{IFR,~then~} F_{w}(x)$ is decreasing in $w$.

A similar proof holds if $F$ is DFR.

Another result concerning total time on test in the case of censored sampling from an IFR distribution may be obtained directly from Theorem 4.6. Simply choose $\bar{A}_{i}=n-i+1, i=1, \cdots, r$, in that theorem. We immediately obtain:

Corollary 5.5. Let $F$ be IFR with mean $\theta$ and $G(t)=1-e^{-t / \theta}$. Then

$$
P_{F}\left[\sum_{1}^{r}(n-i+1)\left(X_{i}-X_{i-1}\right)>x\right] \geqq P_{G}\left[\sum_{1}^{r}(n-i+1)\left(Y_{i}-Y_{i-1}\right)>x\right]
$$

for $x \leqq(n-r+1) \theta$.

Next we consider truncated sampling. If $n$ items are placed on test and successive failure times are observed until a pre-assigned time $t_{0}$, the associated sample is called a truncated sample. Let

$$
V\left(t_{0}\right)=\sum_{i=1}^{r} X_{i}+(n-r) t_{0},
$$

where $r$ denotes the number of observations $\leqq t_{0}$, and is a random variable. 
$V\left(t_{0}\right)$ represents the total time on test up to time $t_{0}$. This statistic occurs in life testing in the exponential case. See, for example, Epstein and Sobel (1955).

THEOREM 5.6. Let $G^{-1} F$ be starshaped on the support of $F, F(0)=0=G(0)$. and $E X=E Y$. Then

$$
E\left[\sum_{1}^{r} X_{i}+(n-r) t_{0}\right] \geqq E\left[\sum_{1}^{s} Y_{i}+(n-s) t_{0}\right],
$$

where $r(s)$ denotes the number of $X(Y)$ observations $\leqq t_{0}$.

Proof. Since $F$ and $G$ have the same mean, they cross at least once. Since $G^{-1} F$ is starshaped, $F$ crosses $G$ exactly once and from below. Hence there exists a least value $x_{0}$ such that $x \geqq G^{-1} F(x)$ for $x \leqq x_{0}$ while $x \leqq G^{-1} F(x)$ for $x>x_{0}$.

Let $Y_{1}^{\prime}=G^{-1} F\left(X_{i}\right)$ and let $s^{\prime}$ denote the number of $Y_{1}^{\prime}, \cdots, Y_{n}^{\prime} \leqq t_{0}$. Then $Y_{1}^{\prime}, \cdots, Y_{n}^{\prime}$ and $s^{\prime}$ have the same joint distribution as $Y_{1}, \cdots, Y_{n}$ and $s$.

(i) Suppose $t_{0} \leqq x_{0}$. Then

$$
\sum_{1}^{r} X_{i}+(n-r) t_{0} \geqq \sum_{1}^{r} Y_{i}^{\prime}+(n-r) t_{0} \geqq \sum_{1}^{s^{\prime}} Y_{i}^{\prime}+\left(n-s^{\prime}\right) t_{0} \text {. }
$$

This implies (5.4).

(ii) Suppose $t_{0}>x_{0}$. Let

$$
\begin{aligned}
Y_{i}^{*} & =Y_{i}^{\prime} & & \text { if } Y_{i}^{\prime} \leqq t_{0} \\
& =t_{0} & & \text { otherwise. }
\end{aligned}
$$

Write

$$
\begin{aligned}
\sum_{1}^{r} X_{i}+(n-r) & t_{0}-\sum_{1}^{s^{\prime}} Y_{i}^{\prime}-\left(n-s^{\prime}\right) t_{0} \\
& =\sum_{1}^{r} X_{i}+(n-r) t_{0}-\sum_{1}^{r} Y_{i}^{*}-(n-r) t_{0} \\
& \geqq \sum_{1}^{r}\left(X_{i}-Y_{i}^{\prime}\right)+\sum_{r+1}^{n}\left(X_{i}-Y_{i}^{\prime}\right),
\end{aligned}
$$

since $X_{i} \leqq Y_{i}^{\prime}$ for $i>r$. Hence

$$
\begin{aligned}
E\left[\sum_{1}^{r} X_{i}+(n-r) t_{0}\right]-E\left[\sum_{1}^{s} Y_{i}+(n-s) t_{0}\right] & \\
& \geqq E \sum_{1}^{n} X_{i}-E \sum_{1}^{n} Y_{\boldsymbol{i}}=0 .
\end{aligned}
$$

REMARK. In the special case in which $G$ is the exponential distribution and $F$ is an IFRA distribution, then $G^{-1} F$ is starshaped. In this case, (5.4) yields a lower bound on the expected total time on test in truncated sampling from an IFRA distribution with known mean.

6. Bounds on expected values of order statistics from monotone failure rate distributions. In Section 3 we obtained explicit bounds on $E X_{i, n}$ assuming $G^{-1} F$ is starshaped [cf. (3.7)]. In particular, if $F$ is IFRA with mean $\theta$, we have the result

$$
\theta \sum_{j=1}^{i}(n-j+1)^{-1} / \sum_{j=1}^{i} j^{-1} \leqq E X_{i, n} \leqq n \theta \sum_{j=1}^{i}(n-j+1)^{-1}
$$

for $1 \leqq i<n$ and $\theta \leqq E X_{n, n} \leqq \theta \sum_{j=1}^{n} Y_{j}$, for $i=n$. The bounds are nontrivial but only sharp for $i=1$ or $i=n$. 
If $F$ is DFRA with mean $\theta$ we have, using (3.6),

$$
\begin{aligned}
0 & \leqq E X_{1, n} \leqq \theta / n, \\
0 & \leqq E X_{i, n} \leqq i \theta \sum_{1}^{i}(n-j+1)^{-1} / \sum_{1}^{i} j^{-1} \text { for } 1<i<n, \\
\theta \sum_{1}^{n} j^{-1} & \leqq E X_{n, n} \leqq n \theta .
\end{aligned}
$$

All lower bounds are sharp. To see this, let

$$
\begin{aligned}
\bar{F}(x) & =0, & & x<0, \\
& =\epsilon e^{-\epsilon x / \theta}, & & x \geqq 0,
\end{aligned}
$$

where $0<\epsilon \leqq 1$. Then $F$ is DFR with mean $\theta$, and for $1 \leqq i \leqq n$,

$$
\begin{aligned}
P\left[X_{i} \geqq x\right] & =\sum_{j=0}^{i-1}\left(\begin{array}{c}
n \\
j
\end{array}\right)[F(x)]^{j}[\tilde{F}(x)]^{n-j} \\
& \leqq \sum_{j=0}^{i-1}\left(\begin{array}{l}
n \\
j
\end{array}\right) \epsilon^{n-j} \exp [-\epsilon x(n-j) / \theta] .
\end{aligned}
$$

Hence $E X_{i}=\int_{0}^{\infty} P\left[X_{i} \geqq x\right] d x<2^{n} \epsilon \theta$. Since we can choose $\epsilon$ arbitrarily close to 0 , we see that $E X_{i} \geqq 0(1 \leqq i<n)$ is sharp. Note that since $\sum_{1}^{n} E X_{i, n}=$ $n \theta, E X_{n, n}$ approaches $n \theta$ as $\epsilon$ decreases to 0 . Hence the upper bound for $i=n$ is also sharp. The upper bound for $i=1$ is attained by the exponential. The other bounds are non-trivial but not sharp.

Using Theorem 4.5 we can obtain additional explicit upper bounds on $\sum_{1}^{n} a_{i} E X_{i}$ assuming $F$ is IFR, $a_{i} \geqq 0$ and $\sum_{1}^{n} a_{i} \leqq 1$.

Theorem 6.1. If $F$ is IFR with mean $\theta, F(0)=0, a_{i} \geqq 0$ for $i=1, \cdots, n$, and $\sum_{1}{ }^{n} a_{i} \leqq 1$, then

$$
\sum_{1}^{n} a_{i} E X_{i} \leqq \theta \sum_{1}^{n} a_{i} E Y_{i} /\left[1-\exp \left(-\sum_{1}^{n} a_{i} E Y_{i}\right)\right]
$$

where $E Y_{i}=\sum_{j=1}^{i}(n-j+1)^{-1}$.

Proof. We may assume without loss of generality that $\theta=1$. As shown in Barlow and Marshall (1964),

$$
\begin{aligned}
F(x ; 1) \geqq b(x ; 1) & =0, & & x \leqq 1, \\
& =1-e^{-w x}, & & x \leqq 1,
\end{aligned}
$$

where $w$ depends on $x$ and satisfies

$$
1-e^{-w x}=w .
$$

Since $F\left[\sum_{1}^{n} a_{i} E X_{i}\right] \leqq 1-\exp \left[-\sum_{1}^{n} a_{i} E Y_{i}\right]$ by Theorem 4.5, we have

$$
b\left[\sum_{1}^{n} a_{i} E X_{i} ; 1\right] \leqq 1-\exp \left[-\sum_{1}^{n} a_{i} E Y_{i}\right] \text {. }
$$

Choose $t$ such that

$$
b(t ; 1)=1-e^{-w t}=1-\exp \left[-\sum_{1}^{n} a_{i} E Y_{i}\right],
$$

where $w$ depends on $t$. It follows that

$$
\sum_{1}^{n} a_{i} E X_{i} \leqq t=\sum_{1}^{n} a_{i} E Y_{i} / w .
$$

Using (6.2) and (6.3) we obtain (6.1). 
Sharp bounds on expected values of order statistics from an IFR distribution can be given in terms of the $p$ th percentile.

TheOREM 6.2. Let $F$ be IFR with $p$ th percentile $\xi_{p}$. Then

$$
E X_{j} \leqq \max \left\{\xi_{p},(p /-\log q)\left[n^{-1}+\cdots+(n-j+1)^{-1}\right]\right\}
$$

and

$$
E X_{j} \geqq \sum_{i=0}^{j-1}\left(\begin{array}{l}
n \\
j
\end{array}\right) \int_{0}^{\xi_{p}}\left(1-e^{x \log q / \xi_{p}}\right)^{i}\left(e^{x \log q / \xi_{p}}\right)^{n-i} d x
$$

where $q=1-p$. All inequalities are sharp.

Proof. To show (6.4), let

$$
\begin{aligned}
G_{\Delta}(x) & =1 & & \text { for } 0 \leqq x \leqq \Delta \\
& =q \exp \left\{\left[\left(x-\xi_{p}\right) /\left(\xi_{p}-\Delta\right)\right] \log q\right\} & & \text { for } x>\Delta .
\end{aligned}
$$

Note that $\bar{G}_{\Delta}(\Delta)=1$ and $\bar{G}_{\Delta}\left(\xi_{p}\right)=q$. Since $\log \bar{F}(x)$ is concave, there exists at least one value of $\Delta \geqq 0$ such that $\bar{G}_{\Delta}(x) \geqq \bar{F}(x)$ for all $x \geqq 0$. Thus $E X_{j} \leqq$ sup $_{0 \leqq \Delta \leqq \xi_{p}} E Y_{j}$ where $Y_{j}$ is the $j$ th order statistic from $G_{\Delta}$. Now

$$
\begin{aligned}
E Y_{j} & =\Delta+\int_{0}^{\infty} \sum_{i=0}^{j-1}\left(\begin{array}{c}
n \\
i
\end{array}\right)\left[G_{\Delta}(x)\right]^{i}\left[\bar{G}_{\Delta}(x)\right]^{n-i} d x \\
& =\Delta+\int_{0}^{\infty}[\Gamma(n+1) / \Gamma(j) \Gamma(n+1-j)] \int_{\sigma_{\Delta}(x)}^{1} t^{j-1}(1-t)^{n-j} d t d x
\end{aligned}
$$

by p. 234, Mood (1950).

To find the maximizing $\Delta$, consider

$$
\begin{aligned}
(\partial / \partial \Delta) E Y_{j} & =1-[\Gamma(n+1) / \Gamma(j) \Gamma(n+1-j)] \int_{\theta_{\Delta}(\Delta)}^{1} t^{j-1}(1-t)^{n-j} d t \\
+ & {[\Gamma(n+1) / \Gamma(j) \Gamma(n+1-j)] \int_{\Delta}^{\infty}\left[G_{\Delta}(x)\right]^{j-1}\left[\bar{G}_{\Delta}(x)\right]^{n-j} q \cdot } \\
& \quad \exp \left\{\left[\left(x-\xi_{p}\right) /\left(\xi_{p}-\Delta\right)\right] \log q\right\}[\log q]\left(x-\xi_{p}\right) /\left(\xi_{p}-\Delta\right)^{2} d x .
\end{aligned}
$$

Since $G_{\Delta}(\Delta)=0,-\left(\xi_{p}-\Delta\right) \partial E Y_{j} / \partial \Delta$ reduces to

$$
\begin{array}{r}
{[\Gamma(n+1) / \Gamma(j) \Gamma(n+1-j)] \int_{\Delta}^{\infty}\left[G_{\Delta}(x)\right]^{j-1}\left[\bar{G}_{\Delta}(x)\right]^{n-j} g_{\Delta}(x)\left(x-\xi_{p}\right) d x} \\
=E Y_{j}-\xi_{p}
\end{array}
$$

where $g_{\Delta}$ is the density of $G_{\Delta}$. Hence

$$
\begin{aligned}
-\left(\xi_{p}-\Delta\right)\left(\partial E Y_{j} / \partial \Delta\right) & \\
& =\Delta-\left[\left(\xi_{p}-\Delta\right) / \log q\right]\left[n^{-1}+\cdots+(n-j+1)^{-1}\right]-\xi_{p} \\
& =-\left(\xi_{p}-\Delta\right)\left\{1+(\log q)^{-1}\left[n^{-1}+\cdots+(n-j+1)^{-1}\right]\right\} .
\end{aligned}
$$

For $j$ such that $1+(\log q)^{-1}\left[n^{-1}+\cdots+(n-j+1)^{-1}\right] \leqq 0$, we have $\partial E Y_{j} /$ $\partial \Delta \leqq 0$. For $j$ such that $1+(\log q)^{-1}\left[n^{-1}+\cdots+(n-j+1)^{-1}\right] \geqq 0$, we have $\partial E Y_{j} / \partial \Delta \geqq 0$. Thus $E Y_{j}$ is maximized in the first case at $\Delta=0$ and in the second case at $\Delta=\xi_{p}$. When $\Delta=0, E Y_{j}=\left(\xi_{p} /-\log q\right)\left[n^{-1}+\cdots+\right.$ $(n-j+1)^{-1}$; when $\Delta=\xi_{p}, E Y_{j}=\xi_{p}$.

To show (6.5), let

$$
\begin{aligned}
\bar{G}(x) & =e^{x \log q / \xi_{p}} & & \text { for } 0 \leqq x<\xi_{p} \\
& =0 & & \text { for } \xi_{p} \leqq x<\infty .
\end{aligned}
$$


$G$ has $p$ th percentile $\xi_{p}$. Moreover, since $\log \bar{F}$ is concave, it cannot cross $\log \bar{G}$ on $\left(0, \xi_{p}\right)$; hence $\bar{G}(x) \leqq \bar{F}(x)$ for all $x \geqq 0$. Thus $E Y_{j} \leqq E X_{j}$, where $Y_{j}$ is the $j$ th order statistic from $G$. But

$$
E Y_{j}=\int_{0}^{\infty} \sum_{i=0}^{n-1}\left(\begin{array}{c}
n \\
i
\end{array}\right)[G(x)]^{i}[\bar{G}(x)]^{n-i} d x .
$$

(6.5) follows from the definition of $G$ given just above. $\square$

7. Properties preserved in taking order statistics from IFR (DFR) distributions. In Barlow and Proschan (1965), pp. 38-39, it is shown that order statistics from an IFR distribution themselves have an IFR distribution. This is not true for spacings from an IFR distribution. To see this, suppose that $F$ is IFR with mass at $a<\infty$. Then the distribution of $X_{2}-X_{1}$ will have a jump at the origin, and hence cannot be IFR. The reverse situation exists for DFR distributions. Order statistics from DFR distributions are not necessarily DFR. This is evident since the exponential is DFR, while the $i$ th order statistic from the exponential is strictly IFR for $i>1$. However, spacings from a DFR distribution are DFR.

Theorem 7.1. If $F$ is DFR, then $X_{i}-X_{i-1}$ has a DFR distribution, $i=1,2$, $\cdots, n$.

Proof. Let $H_{i}$ denote the distribution of $X_{i}-X_{i-1}$. For $i=1, \bar{H}_{1}(x)=$ $[\bar{F}(x)]^{n}$, so that $H_{1}$ is DFR. For $i=2, \cdots, n$, write

$$
\begin{array}{r}
\bar{H}_{i}(x)=[n ! /(i-2) !(n-i+1) !] \int_{0}^{\infty}[F(u)]^{i-2}[\bar{F}(u+x)]^{n-i+1} d F(u) \\
\text { for } i=2, \cdots, n .
\end{array}
$$

Now $\bar{F}(u+x)$ is logarithmically convex in $x \geqq 0$ since $F$ is DFR. Hence, so is $[\bar{F}(u+x)]^{n-i+1}$ and therefore $\bar{H}_{i}(x)$ is, since it is a positively weighted linear combination of logarithmically convex functions [Artin (1931)]. Thus $H_{i}(x)$ is DFR for fixed $i=2, \cdots, n$. $\square$

A stronger property than IFR is the property that $F$ has density $f$ such that $\log f(x)$ is concave where finite; i.e., $f$ is $P F_{2}$. Order statistics do preserve the $P F_{2}$ property, as shown in

Theorem 7.2. Suppose $f$ is $P F_{2}$, with $f(x)$ not necessarily 0 for negative $x$. Then the density $f_{\text {in }}$ of the $i$ th order statistic is also $P F_{2}$ for fixed $i=1,2, \cdots, n$.

Proof. When $f$ is $P F_{2}$, so is $F$ and $\bar{F}$. Thus

$$
f_{i}(x)=[n ! /(i-1) !(n-i) !] F^{i-1}(x) \bar{F}^{n-i}(x) f(x)
$$

is also logarithmically concave. Equivalently, $f$ is $P F_{2}$. $\square$

Theorem 7.3. Let $f$ be $P F_{2}$ with $f(x)$ not necessarily 0 for $x<0$. Then $h_{i}$, the density of $X_{i}-X_{i-1}$ is also $P F_{2}$ for fixed $i=2, \cdots, n$. If $f(x)=0$ for $x<0$, then $h_{1}$ is $P F_{2}$, where $h_{1}$ is the density of $X_{1}$.

Proof. Note

$$
h_{i}(x)=[n ! /(i-2) !(n-i) !] \int_{-\infty}^{\infty} F^{i-2}(u) f(u) f(u+x) \bar{F}^{n-i}(u+x) d u
$$

for $i=2,3, \cdots, n$. Since $f$ is $P F_{2}$, so is $r(u)=F^{i-2}(-u) f(-u)$, 
$s(u)=f(u) F^{n-1}(u)$. Since the $P F_{2}$ property is preserved under convolution,

$$
h_{i}(x)=[n ! /(i-2) !(n-i) !] \int_{-\infty}^{\infty} r(-u) s(u+x) d u
$$

is $P F_{2}$ for fixed $i=2,3, \cdots, n$.

Assuming $f(x)=0$ for $x<0$, we see that $h_{1}$ is $P F_{2}$ from

$$
h_{1}(x)=n f(x) \tilde{F}^{n-1}(x) \text {. }
$$

Acknowledgment. We would like to gratefully acknowledge the help and advice of Albert W. Marshall and Ronald A. Pyke. David W. Walkup helped us formulate Lemma 4.1 and Lemma 4.3.

\section{REFERENCES}

ARTin, E. (1931). Einfuhrung in die Theorie der Gamma Funktion-Hamburger Mathematische Einzelschriften, 11, Heft.

Barlow, R. E., Marshall, A. W., and Proschan, F. (1963). Properties of probability distributions with monotone hazard rate. Ann. Math. Statist. 34 375-389.

Barlow, R. E. and Marshali, A. W. (1964). Bounds for distributions with monotone hazard rate, I. Ann. Math. Statist. 35 1234-1257.

Barlow, R. E. and Proschan, F. (1965). Mathematical Theory of Reliability. Wiley, New York.

Barlow, R. E. and Proschan, F. (1966). Tolerance and confidence limits for classes of distributions based on failure rate. Ann. Math. Statist. 37 1593-1601.

Barlow, R. E. and Proschan, F. (1966). Exponential life test procedures when the distribution has monotone failure rate. Boeing document D1-82-0415. (Submitted to J. Amer. Statist. Assoc.)

Barlow, R. E., Marshall, A. W. and Proschan, F. (In process). Inequalities for starshaped and convex functions.

Beckenbach, E. F. and Bellman, R. (1961). Inequalities. Springer-Verlag, Berlin.

Birnbaum, Z. W., Esary, J. D. and Marshall, A. W. (1965). Stochastic characterization of wear-out for components and systems. Ann. Math. Statist. 37 816-825.

Bruckner, A. M. and Ostrow, E. (1962). Some function classes related to the class of convex functions. Pacific J. Math. 12 1203-1215.

Epstein, B. and Sobei, M. (1953). Life testing. J. Amer. Statist. Assoc. 48 486-502.

Epstein, B. and Sober, M. (1955). Sequential life tests in the exponential case. Ann. Math. Statist. 26 82-93.

Epstein, B. (1960a). Statistical life test acceptance procedures. Technometrics 2 435-446. Epstein, B. (1960b). Estimation from life test data. Technometrics 2 447-454.

HARDY, G. H., LitTlewood, J. E. and Pólya, G. (1929). Some simple inequalities satisfied by convex functions. Messenger Math. 58 145-152.

Hardy, G. H., Littlewood, J. E. and Pólya, G. (1959). Inequalities. Cambridge Univ. Press.

Karamata, J. (1932). Sur une inégalité relative aux fonctions convexes. Publ. Math. Univ. Belgrade 1 145-148.

KARLIN, S. (1964). Total positivity, absorption probabilities and applications. Trans. Amer. Math. Soc. 111 33-107.

Marshall, A. W., Olkin, I. and Proschan, F. (1965). Monotonicity of ratios of means and other applications of majorization. Boeing document D1-82-0490.

Marshall, A. W. and Proschan, F. (1965). An inequality for convex functions involving majorization. J. Math. Anal. Appl. 12 87-90. 
Mood, A. M. (1950). Introduction to the Theory of Statistics (First Edition). McGraw-Hill, New York.

Ostrowski, A. (1952). Sur quelques applications des fonctions convexes et concaves an sens de I. Schur. J. Math. Pure Appl. 31 253-292.

ScHUR, I. (1923). Über eine klasse von mittelbildungen mit anwendungen auf die determinantentheorie. Sitzungsber. d. Berl. Math. Gesellsch. 22 9-20.

van ZWET, W. R. (1964). Convex Transformations of Random Variables. Mathematical Centre, Amsterdam. 